\title{
The Effect of Social Capital, Social Support, Self-Compassion, and Hope Factors Affecting Quality of Life of Breast Cancer Survivors: A Path Analysis Evidence from Surakarta, Central Java
}

\author{
Eka Miftakhul Jannah'), Didik Gunawan Tamtomo'), Bhisma Murti') \\ ${ }^{1)}$ Masters Program in Public Health, Universitas Sebelas Maret \\ 2)Faculty of Medicine, Universitas Sebelas Maret
}

\section{ABSTRACT}

Background: Breast cancer is one of the most common cancers among women in the world. The study aims to analyze the effect of social capital, social support, hope, and self-compassion toward the quality of life of breast cancer survivors.

Subjects and Method: It was a cross sectional study conducted from December 2019 - January 2020. The sample used was 200 breast cancer survivors aged $\geq 18$ years old and were selected by using simple random sampling in Lovely Pink Community Solo. The dependent variable was the symptom and functional aspects of quality of life of breast cancer survivors. The independent variables were sociodemography factor, social capital, social support, hope, and self compassion. Data collection were conducted by using questionnaires. Data analysis was conducted by using path analysis with Stata 13.

Results: Good quality of life among breast cancer survivors increased and was directly affected by symptom aspect with strong social support $(\mathrm{b}=6.63$; $\mathrm{CI} 95 \%=2.52$ up to $10.76 ; \mathrm{p}=$ o.002), high social capital ( $b=3.73$; CI 95\% $=1.17$ up to $6.30 ; \mathrm{p}=0.004)$, high self-compassion $(\mathrm{b}=$ 3.17; CI $95 \%=0.57$ up to $5.77 ; \mathrm{p}=0.017$ ), education $\geq$ high school $(b=4.19 ;$ CI $95 \%=1.41$ up to $6.97 ; \mathrm{p}=0.003)$, age $\geq 50$ years $(b=1.78$; CI $95 \%=0.05$ up to $3.51 ; \mathrm{p}=0.044$ ), and high hope $(b=4.80$; CI 95\%= 1.85 up to $7.75 ; \mathrm{p}=0.001)$. Good quality of life among breast cancer survivors increased and was directly affected by functional aspects with strong social support $(\mathrm{b}=$ 3.13; CI $95 \%=1.61$ up to $4.64 ; \mathrm{p}<0.001$ ), high social capital $(b=1.74$; CI $95 \%=0.31$ up to 3.17 ; $\mathrm{p}=0.017)$, high self-compassion $(\mathrm{b}=1.60 ; \mathrm{CI}$ $95 \%=0.24$ up to $2.94 ; \mathrm{p}=0.021$ ), education $\geq$ high school $(b=2.41$; CI $95 \%=0.95$ up to 3.86 ; $\mathrm{p}=0.001)$, age $\geq 5$ 0 years $(\mathrm{b}=1.38 ; \mathrm{CI} 95 \%=0.11$ up to $2.66 ; \mathrm{p}=0.034)$, and high hope $(\mathrm{b}=2.42 ; \mathrm{CI}$ $95 \%=0.98$ up to $3.86 ; \mathrm{p}=0.001$ ).

Conclusion: Good quality of life among breast cancer survivors in symptom and functional aspects is affected by strong social support, high social capital, high hope, high self-compassion, high education ( $\geq$ high school), and age $\geq 5$ o years old.

Keywords: social support, social capital, hope, self-compassion, quality of life

\section{Correspondence:}

Eka Miftakhul Jannah, Masters Program in Public Health, Universitas Sebelas Maret, Jalan Ir.Sutami 36A, Surakarta, Central Java, Indonesia.Email:ekamiftakhulj@gmail.com. Mobile: o85649665213.

Cite this as:

Jannah EM, Tamtomo DG, Murti B (2020). The Effect of Social Capital, Social Support, Self-Compassion, and Hope Factors Affecting Quality of Life of Breast Cancer Survivors: A Path Analysis Evidence from Surakarta, Central Java. J Epidemiol Public Healt. 5(1): 53-65. https://doi.org/10.26911/jepublichealth.2020.05.01.06

cC) (i) Journal of Epidemiology and Public Health is licensed under a Creative Commons EY NC SA Attribution-Non Commercial-Share Alike 4.0 International License.

\section{BACKGROUND}

Breast cancer is one of the most common cancers among women globally. There were a total of 2,088,849 incidence of breast cancer in 2018 and it claimed 626,679 lives. Within 5 years the global prevalence of breast cancer is 6,875,099 lives and Asia has the highest number that is 2,623,745 cases (Global Cancer Observatory, 2019a). In 2018 breast cancer had the highest incidence compared with other cancers suffered by Indonesia women 
with a total of 58,256 cases. Breast cancer prevalence in Indonesia within 5 years is 160,653 lives (Global Cancer Observatory, 2019b).

Being diagnosed with cancer is a life changing event for most people. At the present time, breast cancer diagnosis is still considered identical to death for the majority of society. The understanding becomes one of the triggers that may disturb the equality of life of patients and their family. Condition as the result of disease process will bring impact toward patients' and families' good quality of life. Quality of life is one of the prognosis factors that affect the survival and death of breast cancer survivors (Nuryati et al., 2017 in Rudiyo et al., 2012).

Quality of life is related to subjective view in the environmental, social, and cultural context. Involving family support network in the treatment session of breast cancer patients is very effective to improve patients' physical, emotional, and mental condition. Therefore, it develops social bonds and relationship network to improve access to social economy sources. The link is called as social capital, in the form of bonds and connections that connect social elements and facilitate plural actions which improve quality of life.

\section{SUBJECTS AND METHOD}

\section{Study Design}

It was analytic observational study with cross sectional approach. The study was conducted in breast cancer survivor's community named Lovely Pink Solo in Surakarta, Central Java, in December 2019 - January 2020.

\section{Population and Sample}

The study population was breast cancer survivor aged $\geq 18$ years old with a total of 200 study subjects. Sample collection was conducted by using simple random sampling.

\section{Study Variables}

Dependent variable of the study was the symptom and functional aspects of the quali- ty of life of breast cancer survivors. The independent variables of the study were age, education, family income, marital status, living with family, having child, hope, social support, self-compassion, and social capital.

\section{Operational Definition of Variables}

Quality of life was breast cancer survivors' perception about their position in life which was measured on symptom and functional aspects at the time. The instrument used was questionnaires EORTC-BR 23 (Sprangers et al., 1996). The continuous data scale was modified into dichotomous to facilitate data analysis. Code $1=$ good quality of life, $\mathrm{O}=$ poor quality of life.

Social support is support obtained from family and friends. The instrument used was multidimensional scale of perceived social support questionnaires (Zimet et al., 2017). The continuous data scale was modified into dichotomous to facilitate data analysis. Code 1=high, $\mathrm{O}=$ low.

Social capital was breast cancer survivors' perception related to good will, friendly feeling, mutual sympathy, close social relationship, and cooperation among breast cancer survivors and their family with a social group. The instrument used was questionnaires. The continuous data scale was modified into dichotomous to facilitate data analysis. Code $1=$ high social capital, $\mathrm{O}=$ low social capital.

Hope was breast cancer survivors' desire for the future of their life. The instruments used was adult hope scale questionnaires (Snyder et al., 1991). The continuous data scale was modified into dichotomous to facilitate data analysis. Code $1=$ high, $\mathrm{O}=$ low.

Self-compassion was affection and mercy to themselves. The instruments used was Self Compassion Scale (SCS)(Raes et al.,2011). The continuous data scale was modified into dichotomous to facilitate data analysis. Code $1=$ high self-compassion, $\mathrm{O}=$ =low self-compassion. 
Jannah et al./ The Effect of Social Capital, Social Support, Self-Compassion, and Hope

Age was breast cancer survivor age at the time of data collection. The instrument used was questionnaires. It used categorical data scale. Code $1=$ age $\geq 50$ years, $O=$ age $<50$ years.

Marital status was breast cancer survivors' nuptial status at the time of data collection, whether they were married/ widow/ unmarried/ not married. The instrument used was questionnaires. It used categorical data scale. Code $1=$ married, $\mathrm{O}=$ widow $/$ unmarried.

Education was breast cancer survivors' last education at the time of data collection. The instrument used was questionnaires. It used categorical data. Code $1=\geq$ High School, $\mathrm{O}=$ $<$ High School.

Income was breast cancer survivors' average monthly salary. The instrument used was questionnaires. It used categorical data. Code $1=\geq$ mean, $\mathrm{O}=<$ mean.

Living with family was breast cancer survivors live in their daily life with nuclear family that consisted of mother (survivor), husband and/or children or live with nonnuclear family. The instrument used was questionnaires. It used categorical data. Code $1=$ live with nuclear family, $\mathrm{O}=$ do not live with nuclear family.

Having children was the history of giving birth and the child was still alive. The instrument used was questionnaires. It used categorical data. Code $1=$ having children, $\mathrm{O}=$ not having children.

\section{Data Analysis}

Univariate analysis was used to describe each dependent variable and independent variable. Bivariate analysis was used to identify the correlation between independent variables and dependent variable by using Chisquare test.

Multivariate analysis explained the effect of independent variables toward dependent variable by using path analysis model. Univariate, bivariate, and multivariate analysis were conducted by using Stata 13 .

\section{Research Ethic}

The study was conducted based on research ethics namely informed consent, anonymity, confidentiality, and eligibility of ethics. Ethical approval of the research was obtained from Health Research Ethics Committee of Dr. Moewardi Regional Hospital, Surakarta, Central Java, Indonesia, No. 1,474/XII/HREC/2019.

\section{RESULTS}

\section{Univariate analysis}

Table 1 indicates the mean value of breast cancer survivors' age was 50.66, the mean value of family income was Rp 2,358,250, the mean value of social capital was 20.99, the mean value of social support was 4.42, mean value of self-compassion was 31.64 , mean value of hope was 23.99.

Mean value of breast cancer survivors' quality of life in symptom aspect was 20.18. Mean value of each indicator in symptom aspect namely side effect was 21.97 , hair loss was 25.50 , arm symptom was 14.75 , and breast symptom was 14.75. Mean value of breast cancer survivors' quality of life in functional aspect was 72.26. Mean of each indicator in functional aspect namely future perspective was 76.67 , sexual function was 61.75, sexual pleasure was 75.33 , and body image was 72.79.

Table 2 indicates breast cancer survivors' quality of life which was divided into 2 aspects namely symptom and functional aspects. There were 140 people (70\%) survivors with good quality of life in symptom aspect and $139(69.50 \%)$ survivors with good quality of life in functional aspect. The characteristics of breast cancer survivors were dominated by age $\geq 50$ years of 137 people (68.50\%), education $\geq$ high school of 161 people (80.50\%), married survivors were 134 people (67\%), with family income $\geq \operatorname{Rp} 2,358,250$ and $<\operatorname{Rp~2,358,250}$ were respectively 100 people (50\%), survivors were dominated live 
Jannah et al./ The Effect of Social Capital, Social Support, Self-Compassion, and Hope

with nuclear family of 159 people (79.50\%), and survivors with children were 172 people (86\%). Breast cancer survivors were dominated by those who had high social capital of 142 people (71\%), obtained strong social support of 143 people (71.50\%), had high hope with a total of 142 people (71\%), and had high self-compassion of 133 people (66.50\%).

2. The result of bivariate analysis

a. Bivariate analysis of variables that affect breast cancer survivors' quality of life in symptom aspect

Table 3 indicates the factors that affect breast cancer survivors' quality of life in symptom aspect, among others are age $\geq 50$ years $(\mathrm{OR}=$ 13.22, $\mathrm{p}<0.001$ ), education $\geq$ High school $(\mathrm{OR}=14.56, \mathrm{p}<0.001)$, income $\geq \mathrm{Rp} 2,358$ ,250 (OR=6.80, $\mathrm{p}<0.001)$, married survivors $(\mathrm{OR}=7.77, \mathrm{p}<0.001)$, survivors who live with nuclear family $(\mathrm{OR}=6.37, \mathrm{p}<0.001)$, survivors with children $(\mathrm{OR}=12.93, \mathrm{p}<0.001)$, high social capital $(\mathrm{OR}=18.08, \mathrm{p}<0.001)$, strong social support $(\mathrm{OR}=47, \mathrm{p}<0.001)$, high hope $(\mathrm{OR}=42.40, \mathrm{p}<0.001)$, and high self-compassion $(\mathrm{OR}=12.23, \mathrm{p}<0.001)$.

\section{Table 1 Description of Variables' Continuous Data}

\begin{tabular}{|c|c|c|c|c|c|}
\hline Variables & $\mathbf{N}$ & Mean & SD & Min. & Max. \\
\hline Age (Year) & 200 & 50.66 & 6.99 & 29 & 73 \\
\hline Income (Rupiah) & 200 & $2,358,250$ & $1,459,183$ & 0 & $15,000,000$ \\
\hline Social Capital & 200 & 20.99 & 7.11 & 5 & 28 \\
\hline Social Support & 200 & 4.42 & 1.52 & 1 & 6 \\
\hline Self-compassion & 200 & 31.64 & 6.29 & 15 & 36 \\
\hline Hope & 200 & 23.99 & 7.09 & 8 & 38 \\
\hline $\begin{array}{l}\text { Breast cancer survivors' quality of } \\
\text { life in symptom aspect }\end{array}$ & 200 & 20.18 & 23.61 & $\mathrm{O}$ & $75 \cdot 50$ \\
\hline \multicolumn{6}{|c|}{ Indicators of quality of life in symptoms aspects based on the EORTC BR23 } \\
\hline Side effect & 200 & 21.97 & 23.26 & o & 80.95 \\
\hline Hair loss & 200 & 25.50 & 31.52 & $\mathrm{O}$ & 100 \\
\hline Arm symptom & 200 & 18.50 & 26.79 & o & 100 \\
\hline Breast symptom & 200 & 14.75 & 20.35 & o & 83.33 \\
\hline $\begin{array}{l}\text { Breast cancer survivors' quality of } \\
\text { life in functional aspect }\end{array}$ & 200 & 72.26 & 28.85 & 6.25 & 100 \\
\hline \multicolumn{6}{|c|}{ Indicators of quality of life in functional aspects based on the EORTC BR23 } \\
\hline Future perspective & 200 & 76.67 & 30.63 & o & 100 \\
\hline Sexual function & 200 & 61.75 & 27.04 & o & 100 \\
\hline Sexual pleasure & 200 & $75 \cdot 33$ & 32.27 & O & 100 \\
\hline Body image & 200 & 72.79 & 34.40 & $\mathrm{O}$ & 100 \\
\hline
\end{tabular}

2. Bivariate analysis of variables that affect breast cancer survivors' quality of life in function aspect

Table 4 indicates the factors that affect breast cancer survivors' quality of life in symptom aspect, among others are age $\geq 5$ o years $(\mathrm{OR}=$ 12.42, $\mathrm{p}<0.001)$, education $\geq$ High School $(\mathrm{OR}=13.98, \mathrm{p}<0.001)$, income $\geq \mathrm{Rp} \mathrm{2,3-}$
$58,250(\mathrm{OR}=5.45, \mathrm{p}<0.001)$, married survivors $(\mathrm{OR}=7.35, \mathrm{p}<0.001)$, survivors who live with nuclear family $(\mathrm{OR}=7.09, \mathrm{p}<0.001)$, survivors with children $(\mathrm{OR}=12.50, \mathrm{p}<$ o.001), high social capital (OR=14.71, $\mathrm{p}<$ o.oo1), strong social support $(\mathrm{OR}=35.68, \mathrm{p}<$ o.o01), high hope $(\mathrm{OR}=32.45, \mathrm{p}<0.001)$, and high self-compassion $(\mathrm{OR}=11.45, \mathrm{p}<0.001)$. 
Jannah et al./ The Effect of Social Capital, Social Support, Self-Compassion, and Hope

Table 2 Description of Variables' categorical data

\begin{tabular}{|c|c|c|c|}
\hline Variables & Criteria & Frequency (n) & $\begin{array}{l}\text { Percentage } \\
\text { (\%) }\end{array}$ \\
\hline \multirow{2}{*}{$\begin{array}{l}\text { Quality of life in symptom } \\
\text { aspect }\end{array}$} & Good & 140 & 70.00 \\
\hline & Poor & 60 & 30.00 \\
\hline \multirow{4}{*}{$\begin{array}{l}\text { Quality of life in functional } \\
\text { aspect } \\
\text { Age }\end{array}$} & Good & 139 & 69.50 \\
\hline & Poor & 61 & 30.50 \\
\hline & $\geq 50$ years & 137 & 68.50 \\
\hline & $<50$ years & 63 & 31.50 \\
\hline \multirow[t]{2}{*}{ Education } & High ( $\geq$ High School) & 161 & 80.50 \\
\hline & Low (<High School) & 39 & 19.50 \\
\hline \multirow[t]{2}{*}{ Marital Status } & Married & 134 & 67.00 \\
\hline & Unmarried/widow & 66 & 33.00 \\
\hline \multirow[t]{2}{*}{ Family Income } & $\geq \operatorname{Rp} 2,358,250$ & 100 & 50.00 \\
\hline & $<\operatorname{Rp} 2,358,250$ & 100 & 50.00 \\
\hline \multirow[t]{2}{*}{ Living with family } & Living with nuclear family & 159 & 79.50 \\
\hline & Not living with nuclear family & 41 & 20.50 \\
\hline \multirow{2}{*}{ Having children } & With children & 172 & 86.00 \\
\hline & Without children & 28 & 14.00 \\
\hline \multirow[t]{2}{*}{ Social capital } & High & 142 & 71.00 \\
\hline & Low & 58 & 29.00 \\
\hline \multirow[t]{2}{*}{ Social support } & Strong & 143 & 71.50 \\
\hline & Weak & 57 & 28.50 \\
\hline \multirow[t]{2}{*}{ Hope } & High & 142 & 71.00 \\
\hline & Low & 58 & 29.00 \\
\hline \multirow{2}{*}{ Self-compassion } & High & 133 & 66.50 \\
\hline & Low & 67 & 33.50 \\
\hline
\end{tabular}

Table 3 The result of chi-square test of variables that affect breast cancer survivors' quality of life in symptom aspect

\begin{tabular}{|c|c|c|c|c|c|c|}
\hline \multirow[t]{3}{*}{ Independent Variables } & \multicolumn{4}{|c|}{ Quality of Life in Symptom Aspect } & \multirow{3}{*}{$\mathbf{O R}$} & \multirow{3}{*}{$\mathbf{P}$} \\
\hline & \multicolumn{2}{|c|}{ Poor } & \multicolumn{2}{|c|}{ Good } & & \\
\hline & $\mathbf{n}$ & $\%$ & $\mathbf{N}$ & $\%$ & & \\
\hline \multicolumn{7}{|l|}{ Age } \\
\hline$<50$ years & 42 & 66.67 & 21 & 33.33 & 13.22 & $<0.001$ \\
\hline$\geq 50$ years & 18 & 13.14 & 119 & 86.86 & & \\
\hline \multicolumn{7}{|l|}{ Maternal last education } \\
\hline Low $(<$ High School $)$ & 30 & 76.92 & 9 & 23.08 & 14.56 & $<0.001$ \\
\hline High ( $\geq$ High School) & 30 & 18.63 & 131 & 81.37 & & \\
\hline \multicolumn{7}{|l|}{ Family Income } \\
\hline$<\operatorname{Rp} 2,358,250$ & 48 & 48.00 & 52 & 52.00 & 6.80 & $<0.001$ \\
\hline$\geq \operatorname{Rp} 2,358,250$ & 12 & 12.00 & 88 & 88.00 & & \\
\hline \multicolumn{7}{|l|}{ Marital status } \\
\hline Unmarried/widow & 39 & 59.09 & 27 & 40.91 & 7.77 & $<0.001$ \\
\hline Married & 21 & 15.67 & 113 & 84.33 & & \\
\hline \multicolumn{7}{|l|}{ Living with nuclear family } \\
\hline Not living with nuclear family & 26 & 63.41 & 15 & 36.59 & 6.37 & $<0.001$ \\
\hline Living with nuclear family & 34 & 21.38 & 125 & 78.62 & & \\
\hline \multicolumn{7}{|l|}{ Having children } \\
\hline Not having & 22 & 78.57 & 6 & 21.43 & 12.93 & $<0.001$ \\
\hline Having & 38 & 22.09 & 134 & 77.91 & & \\
\hline \multicolumn{7}{|l|}{ Social capital } \\
\hline Low $(<20.99)$ & 42 & 72.41 & 16 & 27.59 & 18.08 & $<0.001$ \\
\hline $\operatorname{High}(\geq 20.99)$ & 18 & 12.68 & 124 & 87.32 & & \\
\hline
\end{tabular}




\begin{tabular}{lcccccc} 
Social support & & & & & & \\
Week $(<3)$ & 47 & 82.46 & 10 & 17.54 & 47.00 & $<0.001$ \\
Strong $(\geq 3)$ & 13 & 9.09 & 130 & 90.91 & & \\
Hope & & & & & & \\
Low $(<23.99)$ & 47 & 81.03 & 11 & 18.97 & 42.40 & $<0.001$ \\
High $(\geq 23.99)$ & 13 & 9.15 & 129 & 90.85 & & \\
Self-compassion & & & & & & \\
Low $(<31.64)$ & 43 & 64.18 & 24 & 35.82 & 12.23 & $<0.001$ \\
High $(\geq 31.64)$ & 17 & 12.78 & 116 & 87.22 & & \\
\hline
\end{tabular}

Table 4 The result of chi-square test of variables that affect breast cancer survivors' quality of life in functional aspect

\begin{tabular}{|c|c|c|c|c|c|c|}
\hline \multirow[t]{3}{*}{ Independent Variables } & \multicolumn{4}{|c|}{ Quality of Life } & \multirow{3}{*}{ OR } & \multirow{3}{*}{$\mathbf{P}$} \\
\hline & \multicolumn{2}{|c|}{ Poor } & \multicolumn{2}{|c|}{ Good } & & \\
\hline & $\mathbf{n}$ & $\%$ & $\mathbf{n}$ & $\%$ & & \\
\hline \multicolumn{7}{|l|}{ Age } \\
\hline$<50$ years & 42 & 66.67 & 21 & 33.33 & \multirow[t]{3}{*}{12.42} & \multirow[t]{3}{*}{$<0.001$} \\
\hline$\geq 50$ years & 19 & 13.87 & 118 & 86.13 & & \\
\hline \multicolumn{5}{|l|}{ Maternal last education } & & \\
\hline Low ( $<$ High School) & 30 & 76.92 & 9 & 23.08 & \multirow[t]{2}{*}{13.98} & \multirow[t]{2}{*}{$<0.001$} \\
\hline High ( $\geq$ High School) & 31 & 19.25 & 130 & 80.75 & & \\
\hline \multicolumn{7}{|l|}{ Family Income } \\
\hline$<\operatorname{Rp} 2,358,250$ & 47 & 47.00 & 53 & 53.00 & \multirow[t]{3}{*}{5.45} & \multirow[t]{3}{*}{$<0.001$} \\
\hline$\geq \operatorname{Rp} 2,358,250$ & 14 & 14.00 & 86 & 86.00 & & \\
\hline \multicolumn{5}{|l|}{ Marital status } & & \\
\hline Unmarried/Widow & 39 & 59.09 & 27 & 40.91 & \multirow[t]{3}{*}{$7 \cdot 35$} & \multirow[t]{2}{*}{$<0.001$} \\
\hline Married & 22 & 16.42 & 112 & 83.58 & & \\
\hline \multicolumn{6}{|l|}{ Living with nuclear family } & \\
\hline Not living with nuclear family & 27 & 65.85 & 14 & 34.15 & \multirow[t]{3}{*}{7.09} & \multirow[t]{2}{*}{$<0.001$} \\
\hline Live with nuclear family & 34 & 21.38 & 125 & 78.62 & & \\
\hline \multicolumn{6}{|l|}{ Having children } & \\
\hline Not having & 22 & 78.57 & 6 & 21.43 & \multirow[t]{3}{*}{12.50} & \multirow[t]{2}{*}{$<0.00$} \\
\hline Having & 39 & 22.67 & 133 & 77.33 & & \\
\hline \multicolumn{6}{|l|}{ Social capital } & \\
\hline Low $(<20.99)$ & 41 & 70.69 & 17 & 29.31 & \multirow[t]{3}{*}{14.71} & \multirow[t]{2}{*}{$<0.001$} \\
\hline $\operatorname{High}(\geq 20.99)$ & 20 & 14.08 & 122 & 85.92 & & \\
\hline \multicolumn{6}{|l|}{ Social Support } & \\
\hline Week $(<3)$ & 46 & 80.70 & 11 & 19.30 & \multirow[t]{3}{*}{35.68} & $<0.001$ \\
\hline Strong $(\geq 3)$ & 15 & 10.49 & 128 & 89.51 & & \\
\hline Hoper & & & & & & \\
\hline Low $(<23.99)$ & 46 & 79.31 & 12 & 20.69 & 32.45 & $<0.001$ \\
\hline $\operatorname{High}(\geq 23.99)$ & 15 & 10.56 & 127 & 89.44 & & \\
\hline Self-compassion & & & & & & \\
\hline Low $(<31.64)$ & 43 & 64.18 & 24 & 35.82 & 11.45 & $<0.001$ \\
\hline High $(\geq 31.64)$ & 18 & 13.53 & 115 & 86.47 & & \\
\hline
\end{tabular}

\section{The result of path analysis}

Figure 1 and Table 5 indicate breast cancer survivors' good quality of life increases in symptom aspect with strong social support $(\mathrm{b}=6.63$; CI $95 \%=2.52$ up to $0.76 ; \mathrm{p}=$ o.002), high social capital ( $b=3.73$; $\mathrm{CI} 95 \%=$ 1.17 up to $6.30 ; \mathrm{p}=0.004$ ), high self com- passion $(\mathrm{b}=3.17$; CI 95\%=0.57 up to $5.77 ; \mathrm{p}=$ o.017), education $\geq$ High School $(b=4.19$; CI $95 \%=1.41$ up to $6.97 ; \mathrm{p}=0.003$ ), age $\geq 50$ years $(b=1.78$; CI $95 \%=0.05$ up to $3.51 ; p=$ 0.044), and high hope ( $b=4.80$; CI 95\%= 1.85 up to $7.75 ; \mathrm{p}=0.001)$. 
Figure 2 and Table 6 indicates breast cancer survivors' good quality of life increases in functional aspect with strong social support $(b=3.13$; CI $95 \%=1.61$ up to 4.64 ; $\mathrm{p}<0.001)$, high social capital $(\mathrm{b}=1.74$; CI $95 \%=0.31$ up to $3.17 ; \mathrm{p}=0.017$ ), high selfcompassion $(\mathrm{b}=1.60$; CI 95\%=0.24 up to 2.94; $\mathrm{p}=0.021$ ), education $\geq$ high school $(\mathrm{b}=$ 2.41; CI 95\%=0.95 up to 3.86; $\mathrm{p}=0.001$ ), age $\geq 5$ o years $(\mathrm{b}=1.38$; CI $95 \%=0.11$ up to 2.66 ; $\mathrm{p}=0.034)$, and high hope $(\mathrm{b}=2.42$; CI $95 \%=$ 0.98 up to $3.86 ; \mathrm{p}=0.001$ ). Figure 1 and 2 presented several variables that indirectly affect breast cancer survivors' quality of life in symptom and functional aspects, among others are family income, marital status, having children, and living with family.

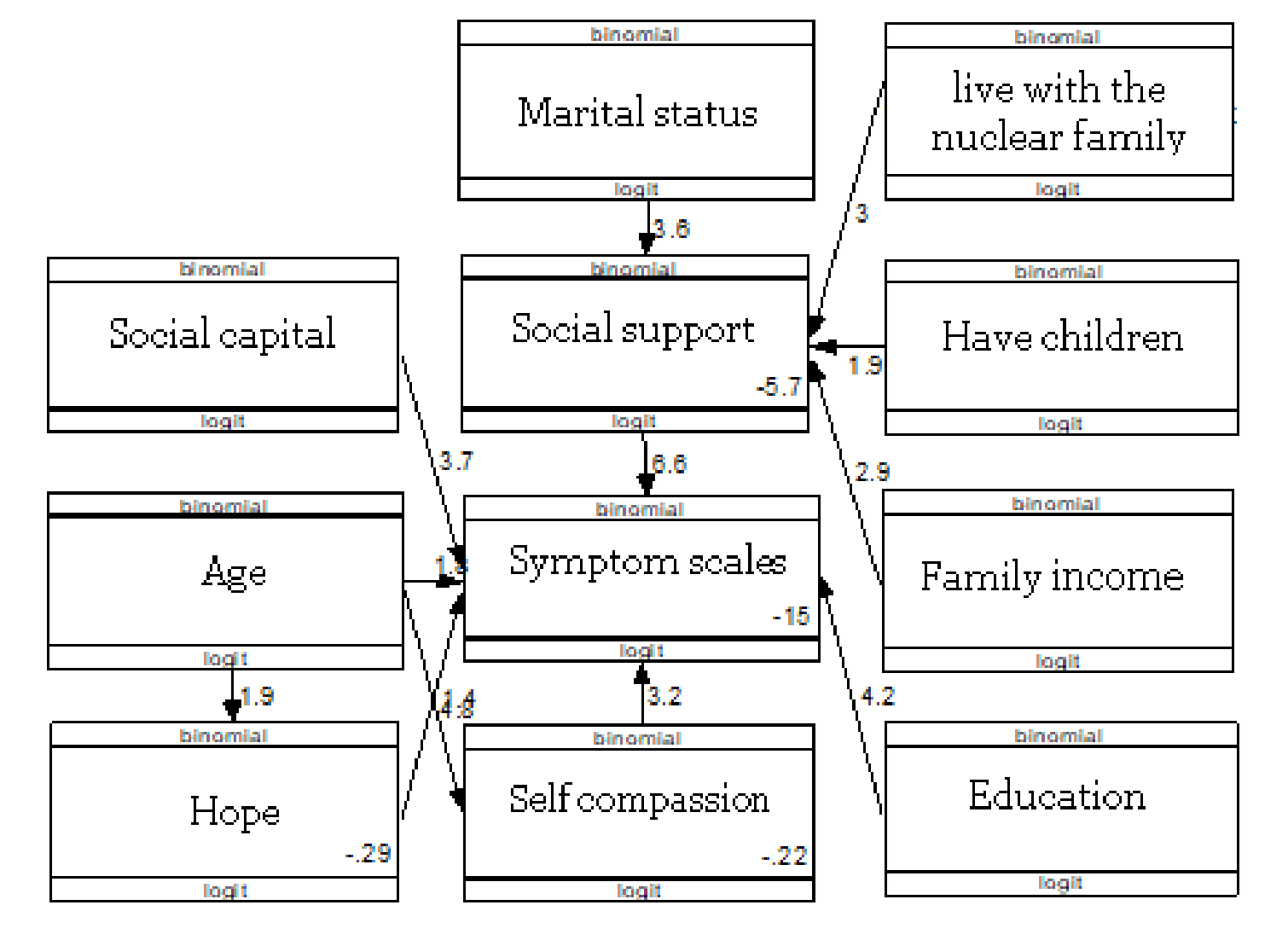

Figure 1 Path analysis model about variables that affect breast cancer survivors' quality of life in symptom aspect 
Jannah et al./ The Effect of Social Capital, Social Support, Self-Compassion, and Hope

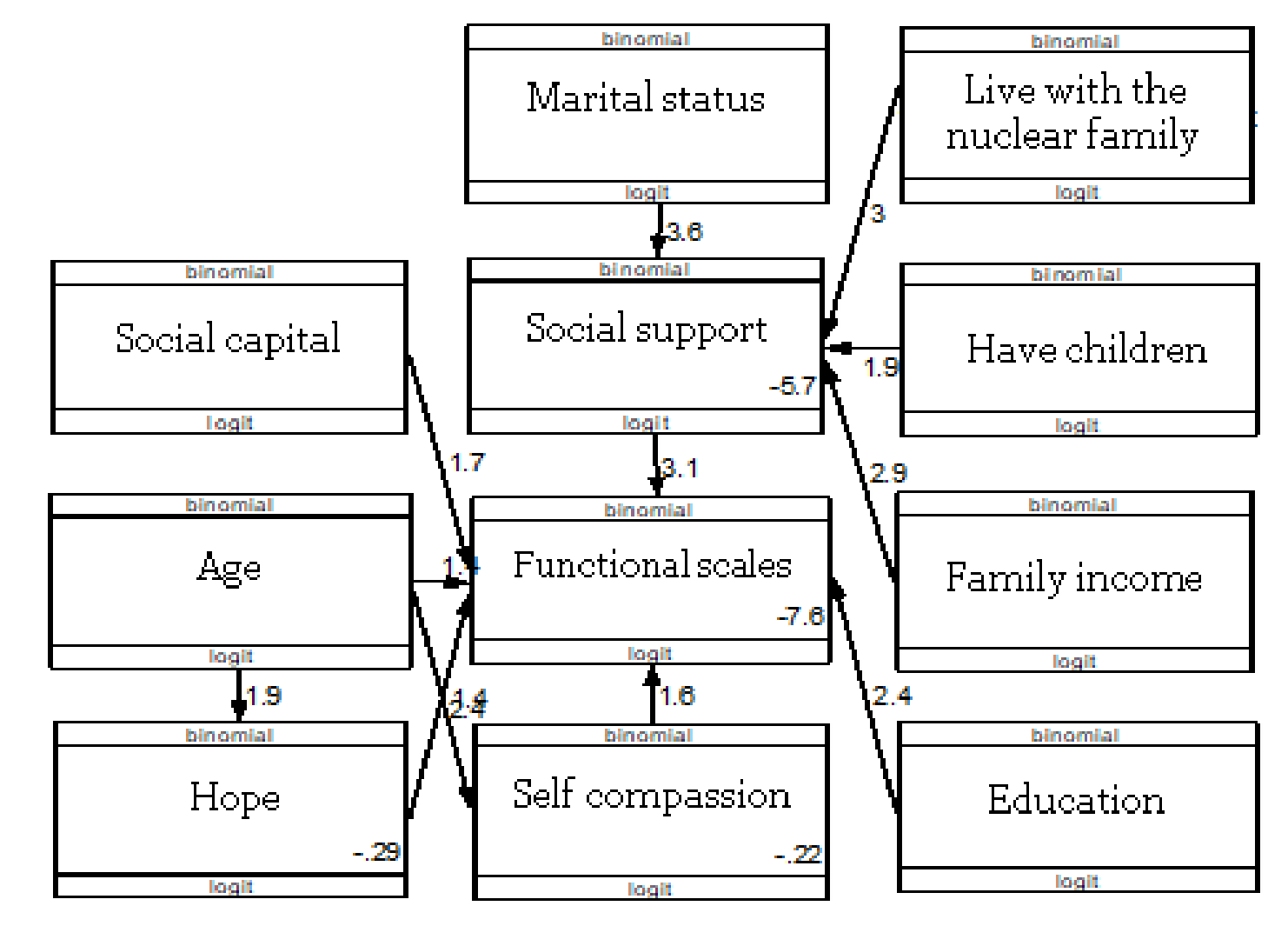

Figure 2 Path analysis model about variables that affect breast cancer survivors' quality of life in functional aspect

Table 5. The path analysis result of independent variable effects toward breast cancer survivors' quality of life in symptom aspect

\begin{tabular}{|c|c|c|c|c|c|}
\hline \multirow{2}{*}{$\begin{array}{l}\text { Dependent } \\
\text { Variables }\end{array}$} & \multirow[t]{2}{*}{ Independent Variables } & \multirow{2}{*}{$\begin{array}{c}\text { Path } \\
\text { Coefficient } \\
\text { (b) }\end{array}$} & \multicolumn{2}{|c|}{ CI 95\% } & \multirow[b]{2}{*}{$\mathbf{p}$} \\
\hline & & & $\begin{array}{l}\text { Lower } \\
\text { Limit }\end{array}$ & $\begin{array}{l}\text { Upper } \\
\text { Limit }\end{array}$ & \\
\hline \multicolumn{6}{|l|}{ Direct effect } \\
\hline \multirow[t]{6}{*}{ Symptom Aspect } & $\leftarrow$ Social Support (Strong) & 6.63 & 2.52 & 10.76 & 0.002 \\
\hline & $\leftarrow \quad$ Social Capital (High) & 3.73 & 1.17 & 6.30 & 0.004 \\
\hline & $\leftarrow$ Self-compassion (High) & 3.17 & 0.57 & 5.77 & 0.017 \\
\hline & $\begin{aligned} \leftarrow \quad & \text { Education } \\
& (\geq \text { High School })\end{aligned}$ & 4.19 & 1.41 & 6.97 & 0.003 \\
\hline & $\leftarrow \quad$ Age $(\geq 50$ years $)$ & 1.78 & 0.05 & $3 \cdot 51$ & 0.044 \\
\hline & $\leftarrow$ Hope (High) & 4.80 & 1.85 & 7.75 & 0.001 \\
\hline \multicolumn{6}{|l|}{ Indirect effect } \\
\hline \multirow[t]{4}{*}{ Social Support } & $\begin{array}{l}\leftarrow \text { Living with nuclear } \\
\text { family (Yes) }\end{array}$ & 3.04 & 1.81 & 4.28 & $<0.001$ \\
\hline & $\leftarrow \quad \begin{array}{l}\text { Family income } \\
(\geq \operatorname{Rp~2,358,250)}\end{array}$ & 2.93 & 1.62 & 4.24 & $<0.001$ \\
\hline & $\leftarrow \quad$ Marital Status (Married) & 3.61 & 2.39 & 4.83 & $<0.001$ \\
\hline & $\leftarrow \quad$ Having children (Yes) & 1.89 & 0.47 & $3 \cdot 31$ & 0.009 \\
\hline Self-compassion & $\leftarrow$ Age $(\geq 5$ o years $)$ & 1.41 & 0.78 & 2.05 & $<0.001$ \\
\hline $\begin{array}{l}\text { Hope } \\
\text { n observation }=200 \\
\text { Log Likelihood }=-2\end{array}$ & $\begin{array}{l}\leftarrow \quad \text { Age }(\geq 50 \text { years }) \\
86.04\end{array}$ & 1.94 & 1.27 & 2.62 & $<0.001$ \\
\hline
\end{tabular}


Jannah et al./ The Effect of Social Capital, Social Support, Self-Compassion, and Hope

Table 6 The path analysis result of independent variable effects toward breast cancer survivors' quality of life in functional aspect

\begin{tabular}{|c|c|c|c|c|c|c|}
\hline \multirow{2}{*}{\multicolumn{2}{|c|}{$\begin{array}{l}\text { Dependent } \\
\text { Variables }\end{array}$}} & \multirow{2}{*}{$\begin{array}{l}\text { Independent } \\
\text { Variables }\end{array}$} & \multirow{2}{*}{$\begin{array}{c}\text { Path } \\
\text { Coefficient } \\
\text { (b) } \\
\end{array}$} & \multicolumn{2}{|c|}{ CI 95\% } & \multirow[b]{2}{*}{$\mathbf{P}$} \\
\hline & & & & $\begin{array}{c}\text { Lower } \\
\text { Limit }\end{array}$ & $\begin{array}{l}\text { Upper } \\
\text { Limit }\end{array}$ & \\
\hline \multicolumn{7}{|l|}{ Direct effect } \\
\hline \multirow[t]{6}{*}{ Functional Effect } & $\leftarrow$ & $\begin{array}{ll}\text { Social } & \text { Support } \\
\text { (Strong) } & \end{array}$ & 3.13 & 1.61 & 4.64 & $<0.001$ \\
\hline & $\leftarrow$ & Social Capital (High) & 1.74 & 0.31 & 3.17 & 0.017 \\
\hline & & $\begin{array}{l}\text { Self-compassion } \\
\text { (High) }\end{array}$ & 1.60 & 0.24 & 2.94 & 0.021 \\
\hline & & $\begin{array}{l}\text { Education } \\
\text { ( } \geq \text { High School) }\end{array}$ & 2.41 & 0.95 & 3.86 & 0.001 \\
\hline & $\leftarrow$ & Age ( $\geq 50$ years) & 1.38 & 0.11 & 2.66 & 0.034 \\
\hline & $\leftarrow$ & Hope (High) & 2.42 & 0.98 & 3.86 & 0.001 \\
\hline \multicolumn{7}{|l|}{ Indirect Effect } \\
\hline \multirow[t]{4}{*}{ Social Support } & $\leftarrow$ & $\begin{array}{l}\text { Living with nuclear } \\
\text { family (Yes) }\end{array}$ & 3.04 & 1.81 & 4.28 & $<0.001$ \\
\hline & & $\begin{array}{l}\text { Family Income } \\
(\geq \text { Rp. } 2,358,250)\end{array}$ & 2.93 & 1.62 & 4.24 & $<0.001$ \\
\hline & & $\begin{array}{ll}\text { Marital } & \text { Status } \\
\text { (Married) } & \end{array}$ & 3.61 & 2.39 & 4.83 & $<0.001$ \\
\hline & $\leftarrow$ & Having children (Yes) & 1.89 & 0.47 & $3 \cdot 31$ & 0.009 \\
\hline Self-compassion & $\leftarrow$ & Age ( $\geq 50$ years $)$ & 1.41 & 0.78 & 2.05 & $<0.000$ \\
\hline $\begin{array}{l}\text { Hope } \\
\text { n observation }=200 \\
\text { Log Likelihood }=-30\end{array}$ & $\leftarrow$ & Age ( $\geq 50$ years $)$ & 1.94 & 1.27 & 2.62 & $<0.001$ \\
\hline
\end{tabular}

\section{DISCUSSION}

1. The effect of age toward breast cancer survivors' quality of life

The result of the study is in line with Sharma and Purkayastha (2017) that younger breast cancer patients (30-39 years) show significantly worse quality of life compare to older age groups in relation with physical functions, social functions, and future perspective. It indicates that younger age group encounters more social barriers during breast cancer treatment, especially post mastectomy compare to older age group.

Younger survivors are still affected by body image, sexual function, hair loss, and future perspective related to economy and family which affect emotion and social activities. Older breast cancer survivors are identified to have better emotional functions and are not concerned about body image (GraellsSans et al., 2018; Sharma et al., 2005 ; and Avis et al., 2005).

\section{The effect of social support toward breast cancer survivors' quality of life}

Decent family harmony and frequent interaction with friends and neighbor, are two specific steps in social support which significantly improve breast cancer patients' quality of life. Social support should be the main component of management and treatment of breast cancer patients (Yan et al., 2016). In this study breast cancer survivors who obtained social support are most likely to have good quality of life in symptom and functional aspects. In addition, it has been found in China that social support is one of the most important factors that affect breast cancer patients' quality of life (Zou et al., 2014 in Zhang et al., 2004 and Fu et al., 2004).

Social support obtained from family and friend alter the survivors to be eager to recover even the probability to completely re- 
cover is very small. The feeling of love, security, and comfort give welfare that may improve breast cancer survivors' quality of life. Support from the fellow breast cancer survivors can also improve breast cancer survivors' quality of life, since they mutually understand their conditions and motivate each other that encourage them to undergo chemotherapy and visit hospital for regular checkups.

\section{The effect of social capital toward breast cancer survivors' quality of life}

The result of the study is in line with Hosseini et al. (2016) who conveyed that social capital has positive correlation toward breast cancer survivors' quality of life. High social capital will improve patients' quality of life and reduce patients' pains.

Correlation between social capital and health may get explained by psychosocial as well as neo materialist theory. Psycho-social theory has revealed that the low level of trust and tenuous social cohesion will turn into negative emotion, subsequently, through a mechanism called psych neuroendocrine. It will generate health disorder. In addition, low level of social capital may stimulate stress and inflict unhealthy behavior, such as smoking habit (Kadarwati et al., 2017 in Pearce and Davey-Smith, 2003). Related with the quality of life of breast cancer survivors who have good adjustment such as able to interact with neighbors as well as people around their residence and participate in the existing activities. Therefore, the feedback of the social capital itself will give good impact toward breast cancer survivors' life for the period of time as well as the future.

\section{The effect of self-compassion to- ward breast cancer survivors' qua- lity of life}

The study is supported by a study from Alizadeh et al. (2018) that conveyed that women with breast cancer who have high self-com- passion indicate less anxiety symptom and high quality of life.

Self-compassion may help people to maintain their health more effectively. High compassion, treating themselves well, and thoughtfulness when they undergo negative events. High self-compassion also improve psychological welfares and endurance against stress (Allen and Leary, 2010; Finlay-Jones et al., 2015).

Laksmi and Widyarini (2018) in Kearney (2017) stated that there is a good correlation between self-compassion and quality of life, in which self-compassion is a good strategy to manage patients' emotion that it will have good impact in their quality of life. People with self-compassion are able to manage stress well so that it may improve body immune system.

\section{The effect of education toward breast cancer survivors' quality of life}

Breast cancer survivors' quality of life is affected by socio demographic factors such as age, level of education, occupation, and marital status (Lusiatun et al., 2016 in Christina, 2011). High level of education teaches human to think more logically and rationally, be able to observe issues from different perspectives to be able to analyze and solve the existing problems well. High level of education will affect women in responding their cancer, including in the effort to find as many as possible information about breast cancer. It is in line with Wardiyah et al. (2014) which stated that breast cancer patients' educational level influence the patients' effort to find information about recovery form breast cancer. The higher the level of education, the higher their ability to find information, and knowledge about certain thing including breast cancer.

\section{The effect of hope toward breast cancer survivors' quality of life}

It was supported by a study by Soylu et al. (2016) which discovered the result of sig- 
Jannah et al./ The Effect of Social Capital, Social Support, Self-Compassion, and Hope

nificantly positive correlation between hope and quality of life among 55 women of breast cancer patients, and a study by Souza and Kamble (2016) which discover significantly positive correlation between hope and quality of life among 397 adult cancer patients. It reflects that the high hope of cancer patients is related with good quality of life. Having positive aspects such as hope, may encourage cancer patients to have endurance in dealing with the disease, which generate better medical outcome.

Patients with high level of hope are less likely to have bad mood, since higher hope is likely to lead cancer patients into more positive thought (Li et al., 2016 in Avey et al., 2014). Li et al. (2016) in Felder et al. (2004) was quoted investigating the existence of positive correlation between hope and treatment's efficacy. Therefore, the higher the hope, the more pains can be tolerated by the breast cancer patients, so that they are able to prevent the decrease in quality of life. Hope is considered as the psychological and spiritual resources which is beneficial to fight cancer (Dreyer and Schwartz-Attias, 2014).

\section{AUTHOR CONTRIBUTION}

Eka Miftakhul Jannah conducted the study, collected data, formulated article, and processed data. Didik Gunawan Tamtomo formulated background of the study and discussion. Bhisma Murti formulated the thinking framework and analyzed the study data.

\section{CONFLICT OF INTEREST}

There was no conflict of interest in the study.

\section{FUNDING AND SPONSORSHIP}

This study was self funded.

\section{ACKNOWLEDGEMENT}

The researchers would like to give their gratitude to the Chairperson and the administrators of Lovely Pink Solo who had given their permission for the study. Our gratitude also goes to all breast cancer survivors who had willingly cooperated as the study subject.

\section{REFERENCE}

Alizadeh S, Khanahmadi S, Vedadhir A, Barjasteh S (2018). The relationship between resilience with self compassion, social support and sense of belonging in women with breast cancer. Asian Pac J Cancer Prev, 19(9): 2469-2474. https://doi.org/10.22034/APJCP.2018.19.9.2469

Allen A, Leary M (2010). Self-compassion, stress, and coping. Soc Personal Psychol Compass, 4(2): 107-118.https://doi.org/doi.org/10.1111/j.17519004.2009.00246.x.

Avis N, Crawford S, Manuel J (2005). Quality of life among younger women with breast cancer. J Clin Oncol, 23(15): 3322-3330.https://doi.org/10.1200/JCO.2005.05.130

Dreyer J, Schwartz-Attias I(2014). Nursing care for adolescents and young adults with cancer: Literature review. Acta Haematol, 132: 363-374. https://doi.org/10.1159/000360213

Finlay-Jones A, Rees C, Kane R (2015). Selfcompassion, emotion regulation and stress among australian psychologists: Testing an emotion regulation model of self-compassion using structural equation modeling. PLoS One, 10(7): 1-19. https://doi.org/10.1371/journal. pone.0133481

Global Cancer Observatory (2019a). Breast Fact Sheets Globocan 2018. Retrieved from http://gco.iarc.fr/today/data/factsheets/cancers/20-Breast-fact-sheet.pdf

Global Cancer Observatory (2019b). Fact Sheets Cancer Indonesia 2018. Retrieved from http://gco.iarc.fr/today/data/factsheets/populations/360-indonesiafact sheets.pdf 
Jannah et al./ The Effect of Social Capital, Social Support, Self-Compassion, and Hope

Graells-Sans A, Serral G, Puigpinós-Riera R (2018). Social inequalities in quality of life in a cohort of women diagnosed with breast cancer in Barcelona (DAMA Cohort). Cancer Epidemiol, 54:38-47. https://doi.org/10.1016/j.canep.2018.03.007

Hosseini S, Mousavi M, Rafiee H, Karimi S (2016). The effect of social capital enhancement on quality of life, treatment compliance and pain in patients with breast cancer. Iran J Cancer Prev, 9(5): 1-9. https://doi.org/10.17795/ijcp4618

Kadarwati, Soemanto R, Murti B (2017). The influence of family support, social capital, self ffficacy, education, employment, income, and residential status on the quality of life among the elderly in Salatiga, Central Java. J Epidemiol Public Health, 02(01): 58-69. https://doi.org/10.26911/jepublichealth.2017.02.01 .06

Kearney K, Hicks R (2017). Self-compassion and breast cancer in 23 cancer respondents: is the way you relate to yourself a factor in disease onset and progress? Psychology, 08: 14-26. https://doi.org/10.4236/psych.2017.81002

Kementerian Kesehatan Republik Indonesia (2015). Pusat Data and Informasi Kementerian Kesehatan Republik Indonesia Situasi Penyakit Kanker.https://www.depkes.go.id/resources/download/pu sdatin/infodatin/infodatin-kanker.pdf

Laksmi A, Widyarini N (2018). Effect of self compassion, perceived social support, self esteem to quality of life on woman affected breast cancer. International Journal of Research Publication, 10(1): 1-12. https://doi.org/10010182018323

Li M, Yang Y, Liu L, Wang L (2016). Effects of social support, hope and resilience on quality of life among Chinese bladder cancer patients: A cross-sectional study. Health Qual Life Outcomes, 14(73): 1-9. https://doi.org/10.1186/s12955-0160481-z

Lusiatun, Mudigdo A, Murti B (2016). The effect of self-efficacy, family support, and socio economic factors on the quality of life of patients with breast cancer at Dr. Moewardi Hospital, Surakarta. J Epidemiol Public Health, 1(3): 182-194. https://doi.org/doi.org/10.26911/jepublichealth.2016.01.03.05.

Nuryati S, Mudigdo A, Murti B (2017). Path analysis on the influence of educational level, stages of cancer, social support, and coping strategy toward the quality of life of breast cancer patients in Dr. Moewardi Hospital, Surakarta. J Epidemiol Public Health, 2(3), 225-235.https://doi.org/doi.org/10.26911/jepublichealth.2017.02.03.04.

Raes F, Pommier E, Neff KD, GuchtDV (2011). Construction and factorial validation of a short form of the self-compassion scale. Clin Psychol Psychother 18: 25055. 10.1002/cpp.702.

Sharma N, Purkayastha A (2017). Factors affecting quality of life in breast cancer patients: A descriptive and cross sectional study eith review of literature. J Mid Life Health, 8(2): 75-83. https://doi.org/10.4103/jmh.JMH

Sharma R, Tobin P, Clarke S (2005). Management of chemotherapy induced nausea, vomiting, oral mucositis, and diarrhoea. Lancet Oncol, 6(2): 93-102. https://doi.org/10.1016/S147020450501735-3

Snyder CR, Harris C, Anderson JR, Holleran SA, Irving LM, Sigmon SX, Gibb June et al(1991). The will and the ways: development and validation of an individual differences measure of hope. J Pers Soc Psychol 6o(4): 570-85. http://dx.doi.org/10.1037/0022-3514.60.4.570

Souza M, Kamble S (2016). Silver lining and hope with the quality of life in adult 
cancer patients. Int $\mathrm{J}$ Indian Psychol, 3(2): 24-31. https://pdfs.semanticscholar.org/ea57/ba6dofa96279be2a57f539e 256fb1651498b.pdf.

Soylu C, Babacan T, Sever A, Altundag K (2016). Patients understanding of treatment goals and disease course and their relationship with optimism, hope, and quality of life: A preliminary study among advanced breast cancer outpatients before receiving palliative treatment. Support Care Cancer, 24(8): 3481-3488. https://doi.org/10.1007/soo520-016-3182-6

Sprangers MAG, Groenvold M, Arraras JI, Franklin J, Velde A, Muller M, Franzini $\mathrm{L}$ et al (1996). The european organization for research and treatment of cancer breast cancer-specific quality-of-life questionnaire module : first results from a three-country field study. J Clin Oncol 14(10): 2756-68. 10.1200/JCO.1996.14.10 .2756 .
Wardiyah A, Afriyanti Y, Budiati T (2014). Identification of factors affecting the breast cancer patients optimism of healing. Jurnal Keperawatan, 5(2): 121-127. https://doi.org/10.22219/jk.v5i2.2341

Yan B, Yang L, Hao L, Yang C, Quan L, Wang $\mathrm{L}, \mathrm{Wu} \mathrm{Z}$ et al. (2016). Determinants of quality of life for breast cancer patients in Shanghai, China. PloS One, 11(4): 114. https://doi.org/10.1371/journal.pone.0153714.

Zimet GD, Dahlem NW, Zimet SG, Farley GK (2017). The multidimensional scale of perceived social support the multidimensional scale of perceived social support. J Pers Assess, 52(1): 30-41. 10.1207/s15327752jpa5201.

Zou Z, Hu J, McCoy T (2014). Quality of life among women with breast cancer living in Wuhan, China. Int J Nurs Stud, 1(1): 79-88. https://doi.org/10.1016/j.ijnss.2014.02.021 